\title{
ИССЛЕДОВАНИЕ ТЕКСТУРНЫХ И МОРФОЛОГИЧЕСКИХ ОСОБЕННОСТЕЙ МУЛЬТИФРАКТАЛЬНОЙ ПОВЕРХНОСТИ МИНЕРАЛОВ С ПРИМЕНЕНИЕМ БИНАРНОГО ПРЕОБРАЗОВАНИЯ
}

\author{
Русанова И.А.
}

Казанский федеральный университет, Казань, irusanova@yandex.ru

\section{Аннотация}

Исследованы возможности применения фрактального анализа и бинарного преобразования для выявления нетипичных областей на цифровых изображениях полевого шпата (трещины, изломы, спайки и структурные изменения). Выделение текстурных неоднородностей и морфологических особенностей проводится при помощи поля фрактальной размерности $D$, поля коэффициента самоподобия $K$ и поля меры $S R G B$ (площади треугольника в координатной системе свойств). Проведённое сегментирование цифровых изображений минералов позволяет оценить области с различными диапазонами значений фрактальных мер и описать сложные мультифрактальные поверхности минералов с учётом спектра фрактальных размерностей, что может быть полезно для прогнозирования измельчения и дробления руд.

\section{Summary}

The possibilities of using fractal analysis and binary transformation for detecting atypical areas on digital images of feldspar (cracks, kinks, adhesions and structural changes) are investigated. The selection of texture heterogeneities and morphological features is carried out using the field of fractal dimension $\mathrm{D}$, the self-similarity coefficient field $\mathrm{K}$, and the measure field SRGB (triangle areas in the coordinate system of properties). The proposed segmentation of digital images of minerals allows one to eliminate the regions with different ranges of fractal measure values and to describe complex multifractal surfaces of minerals taking into account the spectrum of fractal dimensions. This fact which can be used to predict the results of the ores crushing.

\section{Введение}

На сегодняшний день при обработке цифровых изображений большое затруднение вызывает выделение хаотически расположенных небольших объектов и их контуров. Существует множество алгоритмов цифровой обработки изображений. Исследования в данной области по-прежнему актуальны. Разрабатываются новые более точные и эффективные методы обработки изображений, в том числе на основе фрактального анализа, позволяющего исследовать изображения, преобразованные в неоднородную поверхность, например, с показателем яркости по высоте [9, 13], либо цветового кода $[8,11]$. В качестве количественного и объективного критерия описания «шероховатых» нерегулярных текстур может служить фрактальная размерность. Также возможно использование показателя Херста для определения того, является ли поверхностная структура объекта упорядоченной и имеется ли определённая закономерность распределения случайной величины, даже если ряд негауссовский (не является нормально распределённым) $[5,7]$. 
Количественные оценки типоморфных особенностей строения минеральных агрегатов (все элементы текстуры и структуры) позволяют изучать условия их образования и дальнейшего преобразования. На вариативность структурирования поверхности минеральных агрегатов оказывает влияние длительность формирования системы каждого уровня (ионы, зёрна, кристаллы, агрегаты) и срастание границ кристаллов. Степень упорядоченности возникающей системы определяет меру связанности энергии межзёренных границ, от которой зависит прочность минералов. Упорядоченность в зернистой структуре минералов определяется несколькими параметрами, такими как: гранулометрический состав (близкие размеры зёрен), морфология преобладающих кластеров и сбалансированность связанной внутренней энергии системы. Принято считать, что кристаллическая решётка минералов почти идеально связанна и сбалансирована энергетически, несмотря на то, что в реальной решётке, в отличие от её модели, всегда присутствуют дислокации, дефекты и т.д. Наибольшей энергоёмкостью обладают шероховатые грани и извилистые границы зёрен. Степень шероховатости поверхности границы кластера определяется кинетическими параметрами роста зёрен и зависит от степени связанности структуры граничной области, её толщины, скорости релаксации решётки зерна в направлении границы, т.е. от энергии границы. Поверхностная энергия зависит в основном от периметра и шероховатости кластера $[1,4]$. Фрактальная размерность неоднородной поверхности границы минерального кластера демонстрирует чувствительность к количественному описанию сложности структурно-текстурных границ, а также изломов, спайности, твердости и хрупкости поверхности твёрдых тел. Большое количество таких границ приводит к усилению неравномерности измельчения минералов [2, 6, 10].

Проводимое в данной работе исследование может быть полезно для прогнозирования измельчения и дробления руд, раскрытия в процессе дробления минеральных сростков жильных и рудных минералов.

\section{Методы и материалы}

В качестве объектов исследований были взяты цифровые изображения полевого шпата, одного из самых разнообразных, породообразующих минералов магматических, осадочных и метаморфических пород, характеризующихся совершенной спайностью и твёрдостью (рис. 1) [19, 20]. Различные параметры, описывающие форму минеральных зёрен, не всегда дают полную характеристику. Фрактальность не всегда проявляет себя отчётливо, она прячется в хаосе случайной статистики нерегулярных систем. Общей характеристикой таких объектов является пространственная неоднородность с инвариантностью основных геометрических особенностей при изменении масштаба. Количественной мерой распределения неоднородной структуры в пространстве является фрактальная размерность $D$. Исследование таких систем очень интересно, потому что позволяет изучить не только их структуру, но и связь между нею, процессами образования и их свойствами. В основу 
обработки параметров положен алгоритм фрактально-множественного описания экспериментальных данных на основе подхода Мандельброта [13]. Рассмотрим частично упорядоченное конечное множество $A\left(N^{2}\right)$, где $N^{2}-$ число элементов $a_{i j}$ в этом множестве $(i, j=1,2, . . N)$. Будем считать, что элементы множества обладают некоторыми свойствами $H_{\xi}(a)$ (размер, цвет, объём, форма и т.д.), присущими только элементам данного множества. Если общих свойств несколько ( $\xi>1)$, то описание множества должно быть произведено с помощью нескольких фрактальных размерностей.
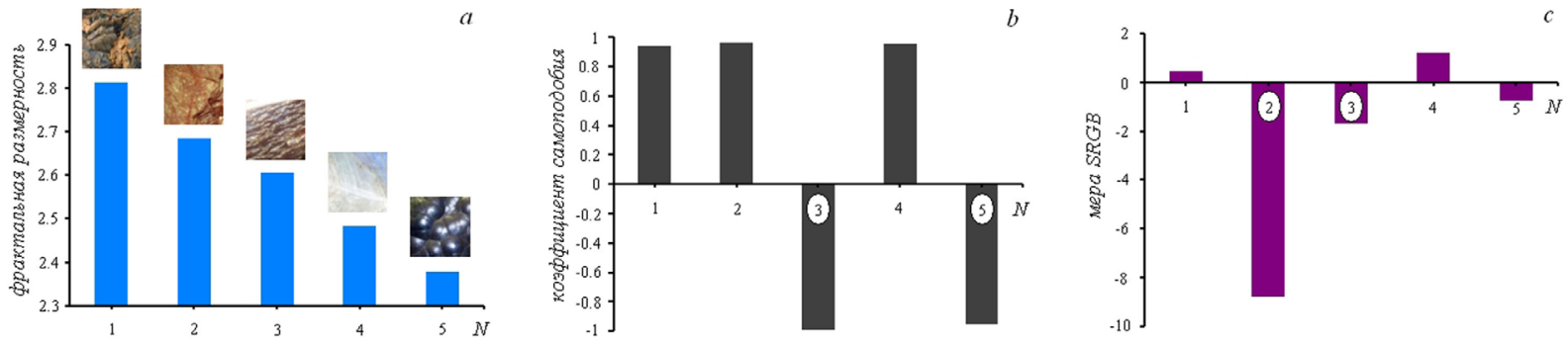

Рис. 1. Гистограммы фрактальной размерности (а), коэффициента самоподобия (b) и меры $S R G B$ (c) для всей поверхности изображения исследуемых образцов минералов (полевого шпата): 1 - колумбит (Ильменские горы Челябинская область), 2 - распространённый вид полевого шпата, 3 - иветные камни Трансбайкальского региона, 4 - «лунный» камень, 5 - гематит (Германия, Саксония).

Определим фрактальную размерность $D$ множества $A\left(N^{2}\right)$ по свойству $H_{\xi}(a)$ угловым коэффициентом зависимости $\log \tilde{A}_{\xi}\left(n^{2}\right)$ от $\log \mathrm{S}_{\xi}\left(n^{2}\right)$, где $\tilde{A}_{\xi}\left(n^{2}\right)$ - число несоприкасающихся поверхностей кубов, покрывающих подмножество, $\mathrm{S}_{\xi}\left(n^{2}\right)$ - площадь, занимаемая элементами подмножества.

$$
D_{\xi}=\sum_{\gamma} \frac{\log \Gamma_{\xi}\left(n_{\gamma+1}^{2}\right)-\log \Gamma_{\xi}\left(n_{\gamma}^{2}\right)}{\left|\log S_{\xi}\left(n_{\gamma+1}^{2}\right)\right|-\left|\log S_{\xi}\left(n_{\gamma}^{2}\right)\right|}\left(\frac{\alpha_{\gamma+1}-\alpha_{\gamma}}{N-1}\right)
$$

Коэффициент самоподобия, позволяющий выявлять минимальные отличия самоподобной структуры от идеального фрактала, определим как $\mathrm{K}_{\xi}=\mathrm{D}_{\xi}^{0} / \mathrm{D}_{\xi}$, где $\mathrm{D}_{\xi}^{0}$ - фрактальная размерность самоподобного множества

$$
D_{\xi}^{0}=\frac{\log \widetilde{A}_{\xi}\left(N^{2}\right)-\log \widetilde{A}_{\xi}(1)}{\left|\log S_{\xi}\left(N^{2}\right)\right|-\left|\log S_{\xi}(1)\right|}
$$

При исследовании фрактальных свойств изображения в качестве свойств $H_{\xi}(a)$ можно выбрать три цвета: красный $(\xi=\mathrm{R})$, зелёный $(\xi=\mathrm{G})$ и синий $(\xi=\mathrm{B})$. Таким образом, описание структуры изображения в данном случае осуществляется тремя фрактальными размерностями $D_{\mathrm{R}}, D_{\mathrm{G}}$ и $D_{\mathrm{B}}$, с помощью которых можно построить некоторую величину $S R G B$ (площадь треугольника в координатной системе свойств), обладающую высокой чувствительностью к изменению структуры изображения $[16,17]$ : 


$$
S R G B=0.5 M \cdot\left[-2\left(D_{\mathrm{R}}+D_{\mathrm{B}}\right)+\left(D_{\mathrm{B}}+D_{\mathrm{G}}\right)+\left(D_{\mathrm{G}}+D_{\mathrm{R}}\right)\right],
$$

где $M$ - масштабный коэффициент. Сложные фракталы, наиболее часто встречающиеся в природе, характеризуются более чем одним показателем скейлинга. Мультифрактальный анализ неоднородного объекта позволяет описать форму минерального кластера таким образом, чтобы каждая из его частей обладала свойствами самоподобия. Для описания мультифракталов необходимо учитывать спектр фрактальных размерностей, так как такие фракталы наряду с геометрическими особенностями обладают определёнными статистическими свойствами $[5,8,11]$.

\section{Результаты}

Фрагменты цифровых изображений исследуемых образцов полевого шпата не подвергались предварительной операции улучшения и имеют размеры $a=b=273$ пикселя. В соответствии с цветовым кодом рабочего пикселя поверхности объекта, в программе производился перевод цветового кода пикселя в высоту точки, с образованием трёхмерного объекта, обладающего неоднородной шероховатой поверхностью. Полученные объекты были исследованы на фрактальность, согласно идеям фрактальной геометрии Мандельброта. Результаты обработки всей поверхности изображения для фрактальной размерности (a), коэффициента самоподобия (b) и меры $S R G B(c)$ представлены на рис. 1. Показано, что наибольшей величиной фрактальной размерности обладает изрезанная, шероховатая поверхность колумбита (рис. 1 a.1), а наименьшим значением - гладкая поверхность гематита (рис. 1 a.5). Нетипичные (аномальные) изменения в структуре поверхности минералов, характеризуемые коэффициентом самоподобия $(K<1))$, присутствуют в образцах цветного камня и гематита (рис. $1 b .3,5$ ). При анализе меры $S R G B$ к последней группе по текстурным признакам добавляется образец 2 (рис. 1 c. 2,3,5).

Дальнейшая обработка изображений минералов проводилось сканированием «окном» размером $a \times b$ пикселей с шагом смещения $s$ (при $s=1$ «окно» является «скользящим», при при $s>1$ «скачущим»). На каждом шаге определялись численные значения фрактальной размерности в «окне» (сегменте), с формированием матрицы и поля фрактальных размерностей (ПФР) (рис. 2) [3,15]. Аналогично были получены поля коэффициента самоподобия $K$ и меры $S R G B$. Выявлено, что в центре области, окружённой сегментами с $D<2.5$, находится сегмент с большей по величине фрактальной размерностью, что возможно связано с изменением степени упорядоченности системы вследствие различной степени связанности структуры граничных областей, ввиду сбалансированности энергии в направлении той или иной границы минерального агрегата (рис. 2 D.I,II). Применим к полученным полям фрактальных мер бинарное преобразование, приводящее к значительному уменьшению большого количества информации, с которой приходится работать, при помощи перевода цветного (или в градиентах серого) изображения $S(x, y)$ в чёрно-белое относительно величины некоторого «порога» 
$S_{\Pi}$, присвоением текущему сегменту изображения значения «1» («чёрный» цвет) либо «0» («белый» цвет или прозрачное «окно» при наложении бинарной маски на изображение), если исходное значение больше (меньше) порогового $[12,18]$. Примем за «пороговое» значение фрактальной размерности величину показателя Херста $H$, для коэффициента самоподобия и меры $S R G B$ численное значение «0». Параметры самоподобия показателя Херста $H$ как и фрактальной размерности представляют собой меры устойчивости статистического явления или меры длительности долгосрочной зависимости стохастического процесса. Бинарное преобразование мультифрактальных поверхностей минералов позволяет выделить ранее визуально скрытые особенности структурно-текстурных границ минеральных кластеров, присущих каждому виду полевого шпата (рис. 2). Показано, что рядом с сегментом, характеризуемым величиной $D \approx 3$, с высокой вероятностью присутствуют сегменты с $2.5<D<2.6$, близкие к значению $D=2.5(H=0.5)$, что является признаком близости к случайной, не фрактальной зависимости. Чем ближе значение показателя $H$ к 1 , тем выше степень устойчивости долгосрочной зависимости стохастического процесса. При $0 \leq H \leq 0.5$ зависимость является
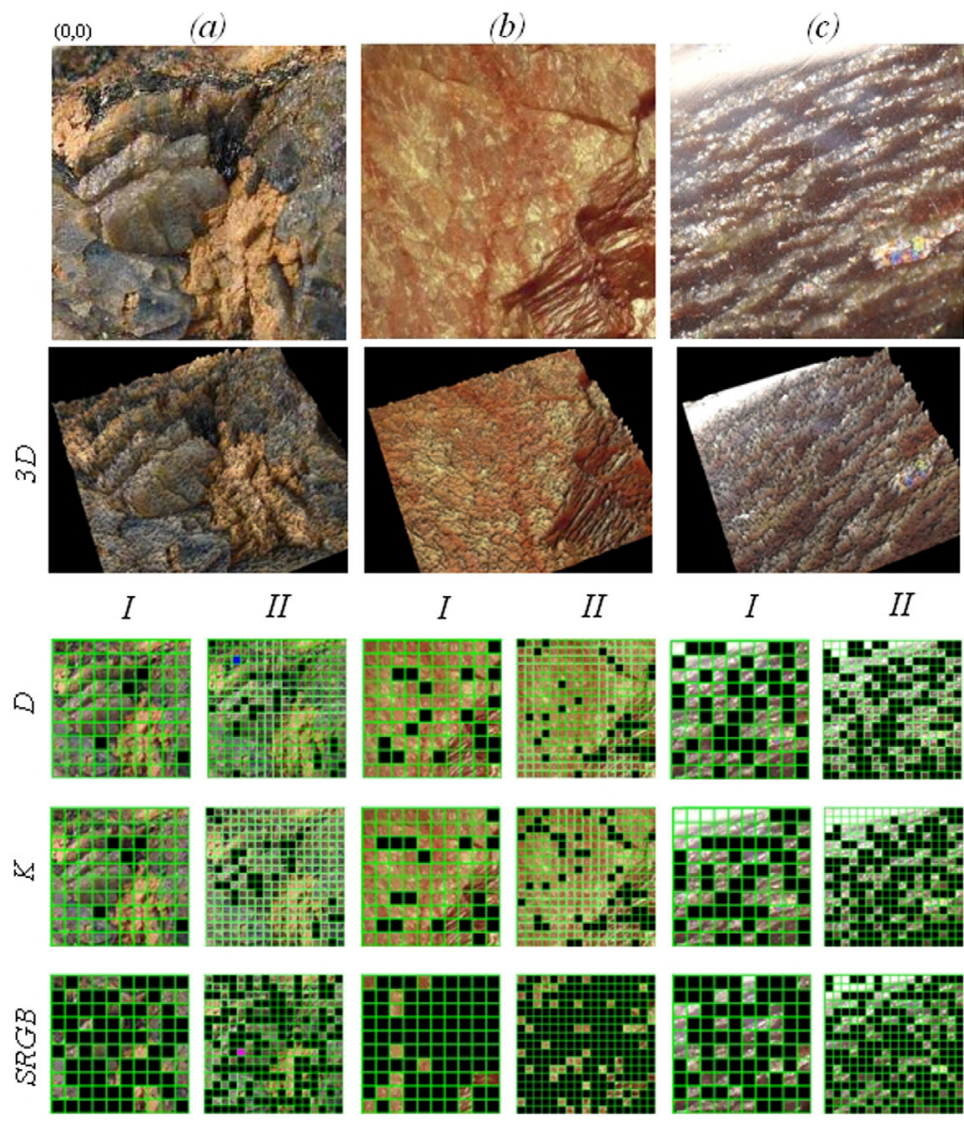

\begin{abstract}
II
\end{abstract}
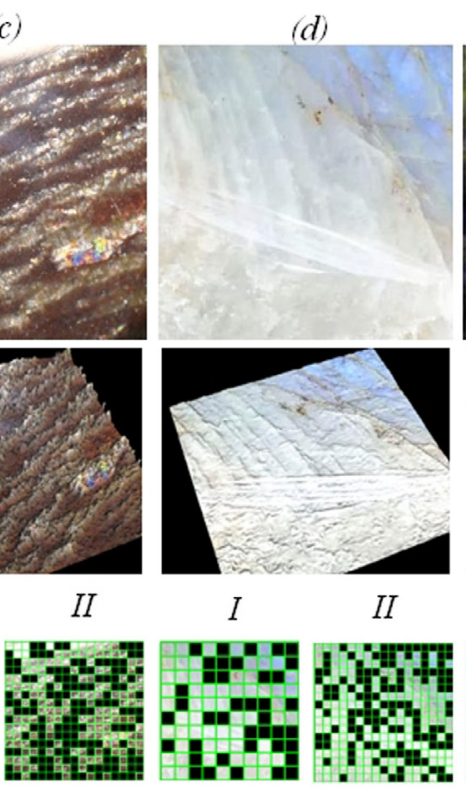

II
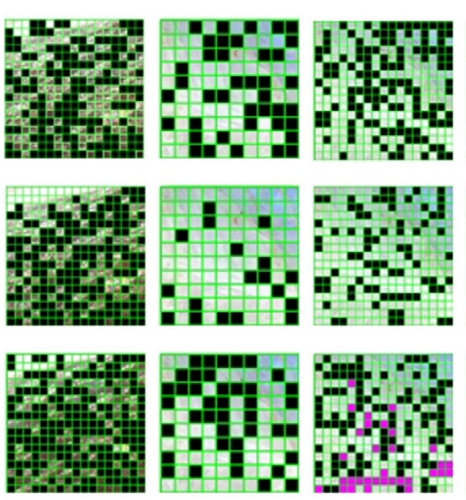

Рис. 2. Фрагменты исследуемых циифровых изображений полевого шпата (а-е), соответствующие им $3 D$ отображения и бинарное преобразование полей фрактальных мер (сканирование «окном» $20 \times 20$ пикселей с $s=7$ (I) и «окном» $16 \times 16$ пикселей с $s=1$ (II)), где «пороговые» значения бинарного преобразования: для поля фрактальной размерности $D 0 \leq H \leq 0.5-« 0 », 0.5<H \leq 1-« 1 »(D>3-« с и н и и ̆ » ~ и в е т)$; для поля коэффищиента самоподобия $K>0-« 0 », K<0-« 1 » ;$ для поля меры $S R G B$ $S R G B>0-« 0 », S R G B<0-« 1 », S R G B=0-«$ розовый» ивет. 
устойчивой, более изменчивой, чем случайный ряд $[5,7]$. Бинарное преобразование поля коэффициента самоподобия увеличивает информативность фрактального анализа, визуально выделяя малейшие нетипичные (аномальные) изменения в структуре поверхности минералов, что возможно связано со степенью связанности структуры граничных областей минеральных кластеров и степенью шероховатости (рис. $2 K$ ).

Бинарное преобразование поля меры $S R G B$ выявляет высокую чувствительность даже к небольшим изменениям в цветовой гамме изображения (рис. $2 S R G B$ ). Уменьшение линейного размера сканирующего «окна» приводит к большей детализации в процессе бинарного преобразования и к более сглаженному частотному распределению гистограмм фрактальной размерности. Дальнейшее уменьшение линейного размера «окна» увеличивает количество сегментов с $D>3$ и областей с $S R G B=0(D(R)=D(G)=D(B))$, что говорит об исчезновении свойств самоподобия в малых геометрических областях.

При исследовании морфологических новообразований не всегда возможно непосредственное выявление природы скрытых механизмов, оказывающих влияние на их развитие. Качественной характеристикой текстурных неоднородностей и морфологических особенностей мультифрактальной поверхности минералов является её структурная взаимосвязанность. Изменение взаимосвязей зачастую позволяет выявить скрытые особенности группировки параметров, в том числе и их взаимные корреляты. Анализ изменения корреляционных связей параметров, их силы и количества, а также выявления присутствия скрытых в их структуре самоподобных свойств рассмотрены в качестве критерия оценки типоморфных особенностей строения минеральных агрегатов. Оценка взаимозависимости исследуемых па-

(a)
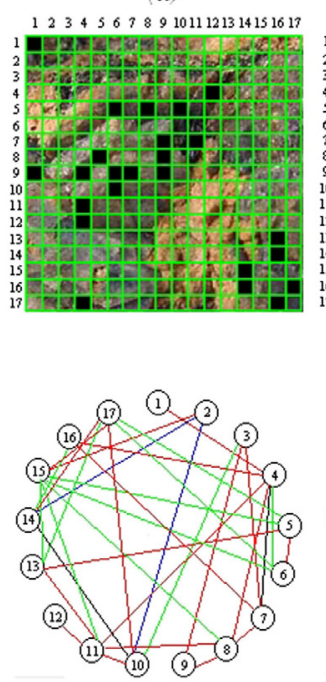
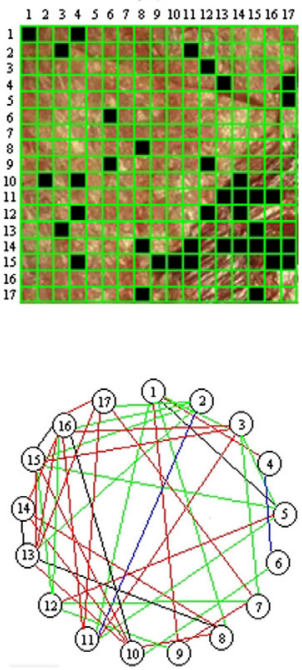

(c)
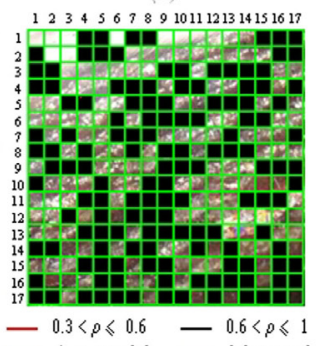

$-0.3<\rho \leqslant 0.6 \quad-0.6<\rho \leqslant 1$
$-1 \leqslant \rho<-0.6 \quad-0.6 \leqslant p<-0.3$

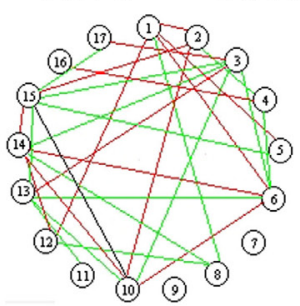

(d)
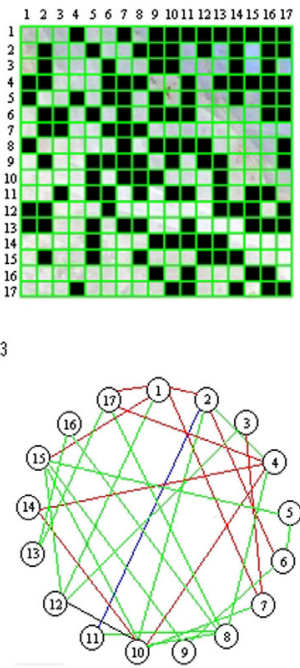

(e)
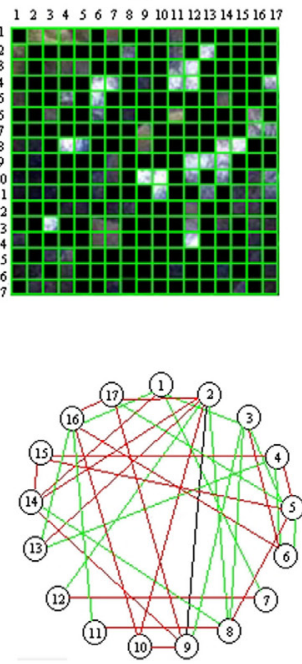

Рис. 3. Бинарное преобразование полей фрактальных размерностей (ПФР) исследуемых иифровых изображений полевого шпата (а-е) (сканирование изображений минералов «окном» $16 \times 16$ пикселей, с s=1) и соответствующие им коррелячионные плеяды исследуемых параметров (вертикальных «слоёв» в структуре поверхности ПФР, где $\rho$ - коэффищиент корреляции). 


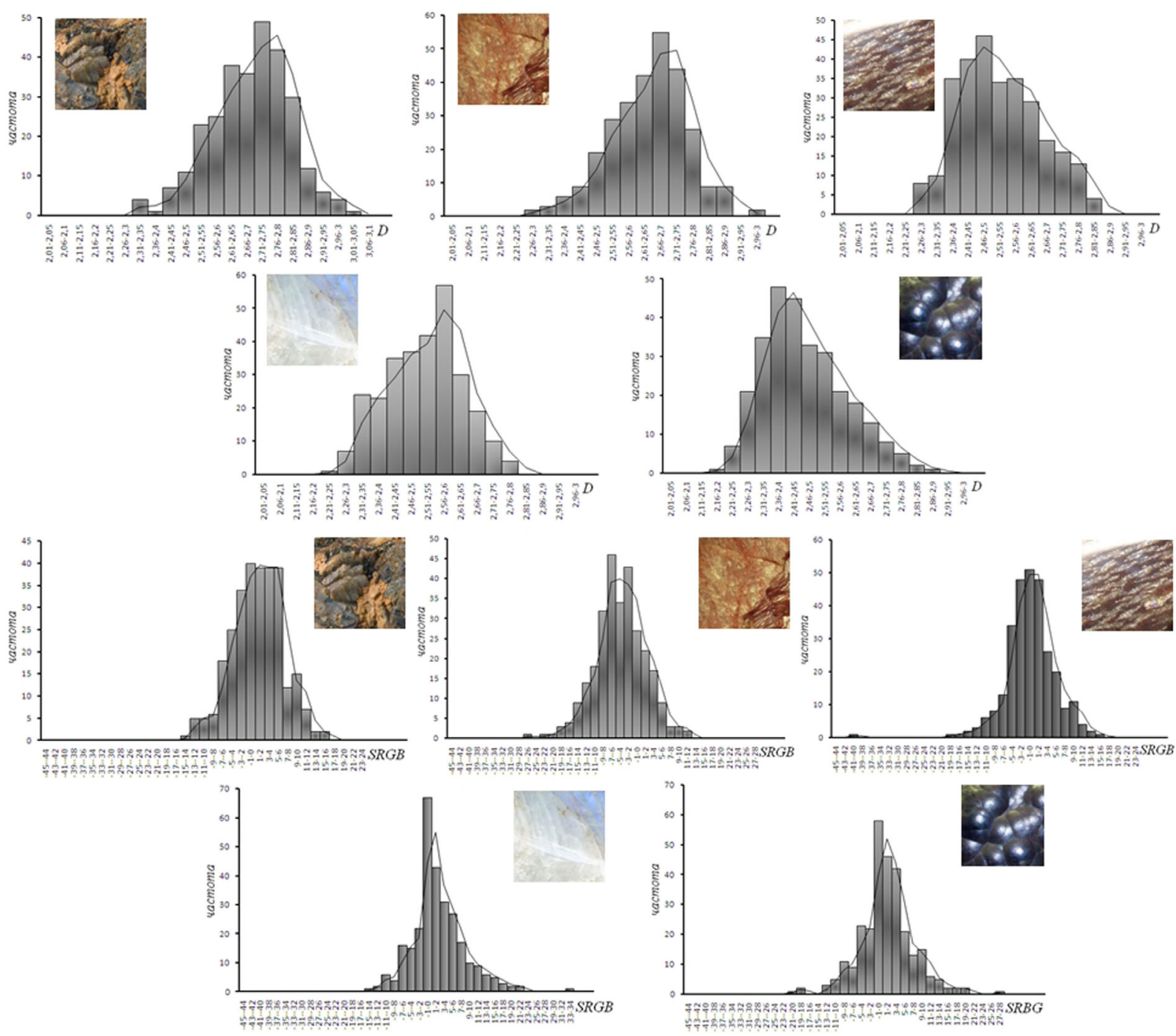

Рис. 4. Гистограммы частотного распределения фрактальной размерности $D u$ меры SRGB (сканирование изображений полевого шпата «окном» $16 \times 16$ пикселей, с шагом смещения $s=1$ ).

раметров ПФР проводилась с помощью корреляционного анализа. Сильная корреляционная связь параметров $\rho$ соответствует значению в пределах от 1 до 0.6 , средняя - от 0.6 до 0.3 и слабая - от 0.3 до 0. В качестве параметров рассматриваются образуемые сканирующим «окном» вертикальные «слои» (1-17) в структуре ПФР поверхности минералов (рис. 3). Присутствие отрицательной корреляции между двумя параметрами указывает на некоторую обратно-пропорциональную связь, позволяющую предположить существование определённого антагонизма между ними [14].

Для различных видов полевого шпата наблюдается выраженное смещение максимумов распределения частоты фрактальной размерности $D$ и различный диапазон интервалов величин меры $S R G B$, с присутствием характерного набора распределения принимаемых значений для разных видов минералов, что может являться паттерном в сравнительной характеристике морфологических и текстурных изменений (трещины, извилистость, поры) (рис. 4). При увеличении размера сканирующего «окна» и выборе «скачущего» режима $(s>1)$ наблюдается потеря детализации визуально выделяемых 
особенностей неоднородной поверхности минералов, с сохранением характерного спектра распределения фрактальной размерности, с потерей сглаженности частотного распределения.

\section{Заключение}

Показано, что поверхностная структура полевого шпата с неоднородными включениями (микротрещины, изломы и т.п.) имеет выраженные фрактальные свойства. Выявлена чувствительность бинарного преобразования полей фрактальных мер к количественному описанию типоморфных особенностей структурно-текстурных границ мультифрактальных минеральных агрегатов, позволяя проводить количественное описание, идентифицирование и прогнозирование усиления неравномерности в процессе измельчения и дробления минералов.

\section{Список литературы}

1. Бродская Р.Л., Марин Ю.Б. Факторы агрегации и структурирования минерального вещества и генетическая интерпретация строения минеральных агрегатов. 2011. Вестник. 5. С. 2-6.

2. Горяинов П.М., Иванюк Г.Ю. Фрактальные свойства месторождений железистых кварцитов - ресурс новых знаний о серед их зарождения. Синергетические прицнипы геоогического анализа. Воронеж: Вестник ВГУ. 2012. № 2. С. 23-31.

3. Грушенко М.В., Пащенко Р.Э., Петрушенко Н.Н., Шаповалов А.В. Обработка аэрокосмических изображений с использованием поля фрактальных размерностей. Системы обработки информации. 1. 2006. С. 35-42.

4. Дымков Ю.М. Минеральные индивиды и минеральные агрегаты / Генезис минеральных индивидов и агрегатов. М: Наука. 1966.

5. Завгородний А.Б. Современные методы исследования структурноинформационных свойств естественных систем // Электроника и связь. № 1. 2010. С. 60-65.

6. Изоитко В.М., Бродская Р.Л., Баданина И.С., Петров С.В. Исследование типоморфных и технологических свойств шеелита месторождения Катлар с помощью стереометрического анализа // Обогащение руд. № 3 (1). 1995. С. 27-32. 7. Кириченко Л.О. Сравнительный анализ статистических свойств оценок показателя Херства // Вестник Нац. техн. ун-та ХПИ. № 21. 2010. С. 88-95. 8. Кульков С.Н., Томаш Ян, Буяков С.П. Фрактальная размерность поверхностей пористых керамических материалов // Письма в ЖТФ. 2006. Т. 32. Вып. 2. С. 51-55.

9. Павлов А.Н., Анищенко В.С. Мультифрактальный анализ сложных систем. Успехи физических наук. 2007. № 177 (8). С. 859-876.

10. Петров Д. Записки Горного института. 2003. № 1. С. 28.

11. Потапов А.А., Булавкин В.В., Герман В.А., Вячеславова О.Ф. Исследование микрорельефа обработанных поверхностей с помощью методов фрактальных сигнатур // Журнал технической физики. 2005. Т. 75. № 5. С. 28-45. 
12. Сальников И.И. Информационная оценка изображений в системах технического зрения // Искусственный интеллект. 2011. № 3. С. 261-270.

13. Федер Е. Фракталы. М: Мир. 1991. 254 с.

14. Харман Г. Современный факторный анализ. М.: Статистика. 1972. 484 с. 15. Яцевич С.Е., Иванов В.К., Пащенко Р.Э. Обработка радиолокационных изображений сельскохозяйственных полей с помощью фрактального анализа // Современные проблемы дистанционного зондирования Земли из космоса. 2008. Вып. 5. № 1. С. 194-200.

16. Rusanova I.A. Study of Fractal Structure of Images of Nail Bed and Periungual Epithelium // Biomedical Engineering. 2013. V. 47. N 2. P. 83-85.

17. Tayurskii D.A., Rusanova I.A. The Fractal Analysis of the Images and Signals in Medical Diagnostics / Fractal Analysis, Applications in Health Sciences and Social Sciences. 2017. Ch. 3. P. 57-86.

18. Xianyu Jin, Bei Li, Ye Tian, et.al. Study on Fractal Characteristics of Cracks and Pore Structure of Concrete based on Digital Image Technology // Research Journal of Applied Sciences, Engineering and Technology. 2013. V. 5. N. 11. P. 3165-3171. 19. Полевой шпат и другие минералы: [сайт] URL: http://fb.ru/article/67606/ polevoy-shpat-i-drugie-mineralyi

20. Фотогаллерея минералов [сайт] URL: http://klopotow.narod.ru/mineral/ gallery/index.htm 